\title{
INVESTIGATION OF RESONANCE CURVE WITH RESPECT \\ TO VARIATION OF CAPACITY
}

\section{by}

\section{LEO EVERETT HUDIBURG}

B. S., Kansas State Teachers College,Pittsburg,1923

\section{A THESIS}

submitted in partial fulfillment of the requirements

$$
\begin{aligned}
& \text { for the degree of } \\
& \text { MASTER OF SCIENCE }
\end{aligned}
$$

KANSAS STATE AGRI CULTURAL COLLEGE 
TABLE OF CONTENTS

$\underline{\text { Page }}$

ABSTRACT -. . . . . . . . . . . . 3

INTRODUCTION • • • • . . . . . . 4

THEORY . . . . . . . . . . 7

CONSTRUCTION OF APPARATUS . . . . 28

RESULTS AND CONCLUSIONS . . . . . 43

ACKNOWLEDGMENT • . . . . . . . 54

SUPPLEMENTARY READING . • • • • . 55 
INVESTIGATION OF RESONANCE CURVE WITH RESPECT

TO VARIATION OF CAPACITY

\section{ABSTRACT}

An investigation of the possibility of using a vibrating variable condenser in a Hartley Oscillator circuit to modulate the radio-frequency of the circuit, and a study of the effect of using the same type of condenser in a receiving circuit to modulate its radio-frequency.

A receiving set in the form of a wave meter containing a variable condenser that may be made to vibrate at voice frequency is coupled inductively to a Hartley Oscillator and tuned to its frequency. It is found that the current in the wave meter fluctuates with the capacity change and that $I^{2} \alpha \frac{1}{C_{1}-C_{2}} \cdot$ This shows that the effective current in an oscillatory circuit of extremely low resistance and operating near the peak of the resonance curve, may be controlled by the application of an audio-frequency variation of capacity.

These results are, in form, very similar to those obtained with respect to the characteristic curve of a vacuum tube when changing the grid voltage. This makes 
possible a system of modulation in which the radio-frequency changes according to the action of an audio-frequency control, but without change of amplitude, and without direct observance of the usual heterodyning rules of audio and radio frequencies. It is different from the ordinary type of modulation in which the audio-frequency is impressed upon the carrier wave as an envelope of the amplitude of the latter, and with the ordinary heterodyning rule of the addition and subtraction of the audio and radio frequencies.

\section{INTRODUCTION}

At the present time the vacuum tube is the only means of modulation and amplification that has proved successful for work in radio telephony. With this in mind, it was decided to investigate the possibilities of a mechanical modulator operating by small variation of capacity in one of two coupled radio circuits.

When the three-electrode vacuum tube is used as an amplifier and the plate current is plotted as ordinates against the grid voltage, a characteristic curve is obtained. (See Plate I.) The useful portion of this curve lies between the points $\underline{a}$ and $\underline{b}$. 
The resonance curve obtained by plotting the current against the capacity in an oscillating circuit of very low resistance: bears a certain resemblance to the tube characteristic curve of Plate I.

When one-half of this resonance curve is compared with that of the useful portion of the tube curve, it is seen that they are very similar. This suggests the possibility of placing a vibrating condenser in the circuit of an oscillator and obtaining effects of modulation and amplification analogous to those ordinarily obtained with the use of a tube. In other words, the construction is of a mechanical modulator and, in some cases, amplifier to replace the vacuum tube.

The object of this work was to determine (1) what effect a vibrating condenser, placed in a receiving circuit coupled with an oscillator, would have on the current in the circuit; and (2) what effect the vibrating condenser placed in the oscillator circuit would have upon the coupled receiving circuit. 


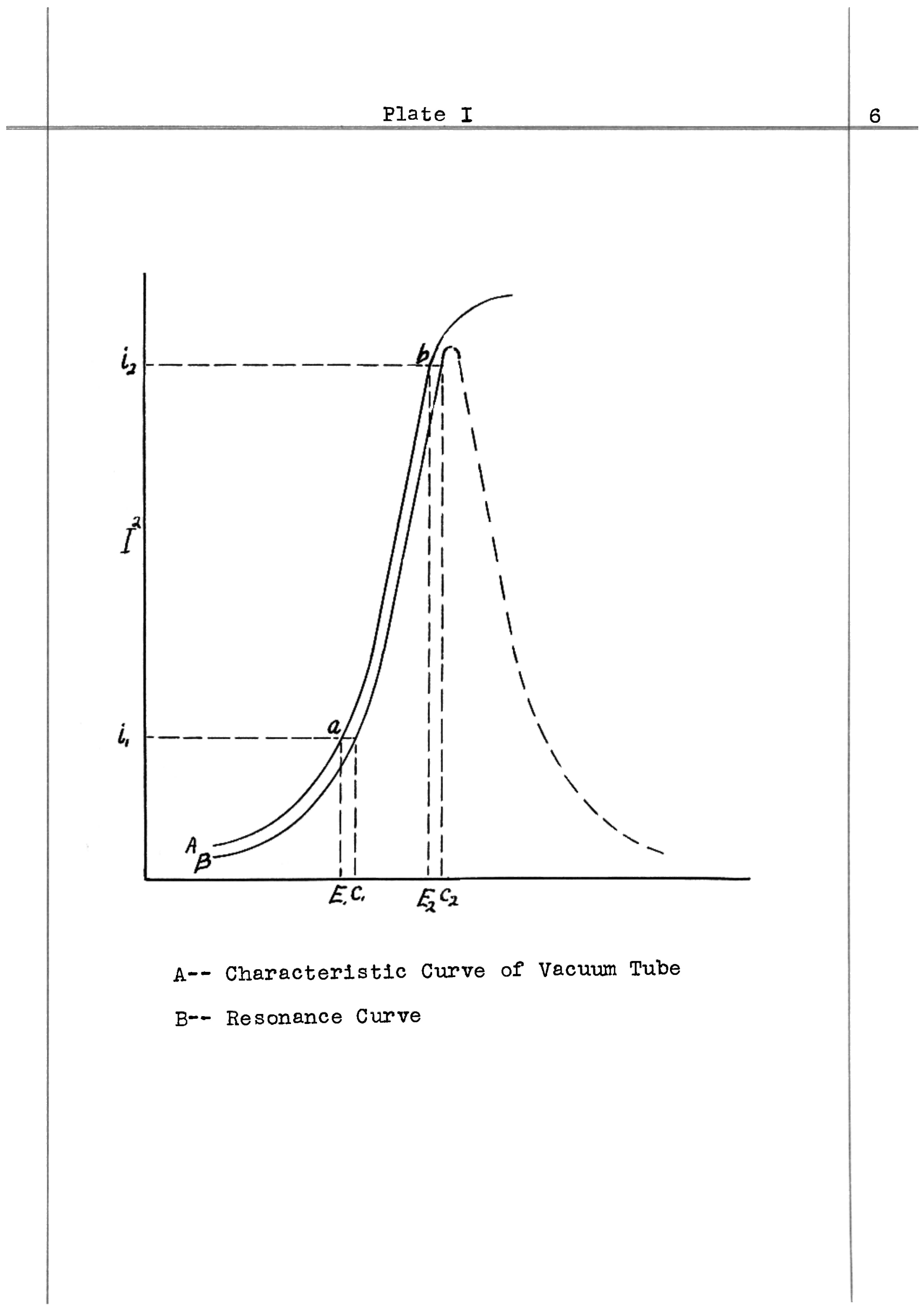




\section{THEORY}

From the wiring diagram it is seen that each circuit used consists of inductance, capacity, and resistance in series, with an oscillating current flowing through them. Therefore we will consider what happens in such a circuit under the existing conditions.

"Circuit containing constant resistance, constant selfinductance and constant capacitance in series."

The solutions for the above conditions are taken from the "Principles of Alternating Current" by Lawrence as given on pages 150 to 168 , as follows.

The conditions in a circuit containing constant resistance, constant self-inductance, and constant capacitance in series are completely determined by the equation

$$
e=r i+L \frac{d i}{d t}+\frac{q}{c}
$$

where $e$ is the impressed electromotive force drop and ri, I $\frac{d i}{d t}$ and $\frac{q}{c}$ are, respectively, the component of the impressed electromotive force drop to supply the ohmic drop, the drop due to self-induction and the drop due to the condenser. If the electromotive force, $e$, is in volts, $r$ must 
be in ohms, I in henrys, and $\mathrm{C}$ in farads. The current and charge will then be in amperes and coulombs, respectively. The energy relation corresponding to equation (1) is $\int_{0}^{T} e i d t=\int_{0}^{T} r i^{2} d t+\int_{0}^{T} L \frac{d i}{d t} i d t+\int_{0}^{T} \frac{q}{c} i d t$ 88

The first term of the second member of equation (2) is the energy dissipated in heat in the resistance of the circuit. The second term is the energy stored in the magnetic field of the inductance and the third term is the energy stored in the electrostatic field of the condenser.

The solution of equation (1) for current in terms of $e, r, L, C$, and $t$ will evidently depend for its form upon $\underline{e} \cdot$ The case involved in this problem is "A Simple Harmonic Electromotive Force Impressed on a Circuit Containing Constant Resistance, Constant Self-inductance and Constant Capacitance in Series." In this case the impressed electromotive force is of the form

$$
e=E_{m} \sin (W t+\mathcal{L}) \text { and }
$$

$$
\mathrm{E}_{\mathrm{m}} \sin (W t+\mathcal{C})=r i+\mathrm{L} \frac{\mathrm{di}}{\mathrm{dt}}+\frac{\mathrm{q}}{\mathrm{C}}
$$

129

or $\mathrm{E}_{\mathrm{m}} \sin (w t+\mathcal{L})=r \frac{\mathrm{dq}}{\mathrm{dt}}+\mathrm{L} \frac{\mathrm{d}^{2} \mathrm{q}}{\mathrm{d} \mathrm{t}^{2}}+\frac{\mathrm{q}}{\mathrm{c}}$ 
The complete solution of this linear differential equation of the second order, the first term of which is a function of $t$, is the sum of the transient and the steady value of the charge. The solution is of the form

$$
\mathrm{q}=\mathrm{Y}+\mathrm{u}
$$

When $Y$ is the transient term, 1.e., the complementary function of the differential equation, and $\underline{u}$ is the particular integral and represents the steady state, the transient term or complementary function is found by putting the first term, $\mathrm{F}_{\mathrm{m}} \sin (w t+\mathcal{L})$, equal to zero. The value of the complementary function evidently depends on the relation of 2

$r \mathrm{C}$ to 4I. Its value is of the form

$$
\begin{aligned}
& q=A_{1} \epsilon^{a_{1} t}+A_{2} \epsilon^{a{ }^{2} t} \\
& i=A_{1} a_{1} \epsilon^{a_{1} t}+A_{2} a_{2} \epsilon^{a_{2} t}
\end{aligned}
$$

It is oscillatory when $r^{2} c$ is less than $4 L$ or $r$ is less than $2 \sqrt{\frac{L}{C}}$, the frequency being given by

$$
f=\frac{1}{T}=\frac{\sqrt{1-\frac{r^{2} c}{4 L}}}{2 \pi \sqrt{L C}}
$$

which is determined by the constants of the circuit. The frequency is entirely independent of the frequency of the 
impressed voltage. The constants for the transient terms for charge and current when the impressed voltage is sinusoidal are determined from the charge and the current when $t=0$. When the transient terms are oscillatory, the constants are determined from the equations

$\left.q=\epsilon^{a t} f A_{1}(\operatorname{Cos} b t+j \sin b t)+A(\operatorname{Cos} b t-j \sin b t)\right\}+E C$ $=\left(A_{1}+A_{2}\right) \epsilon^{a t} \operatorname{Cos} b t+j\left(A_{1}-A_{2}\right)^{a t} \sin b t+E C$ $=A \epsilon^{a t} \cos b t+B \epsilon^{a t} \sin b t+E C \quad 106$

in which $A=\left(A_{1}+A_{2}\right)$ and $B=j\left(A_{1}-A_{2}\right)$

From equation (6), since $i=\frac{d q}{d t}$

$i=(A a+B b) \epsilon^{a t} \operatorname{Cos} b t+(B a-A b) \epsilon^{a t} \sin b t$ (7) 107 if $t=0 ; q=0$ and $i=0$

Therefore, $\quad A=-E C$

$$
B=-\frac{a}{b} A={\frac{r C}{\sqrt{4 L C-r^{2} c^{2}}}}^{B C}
$$




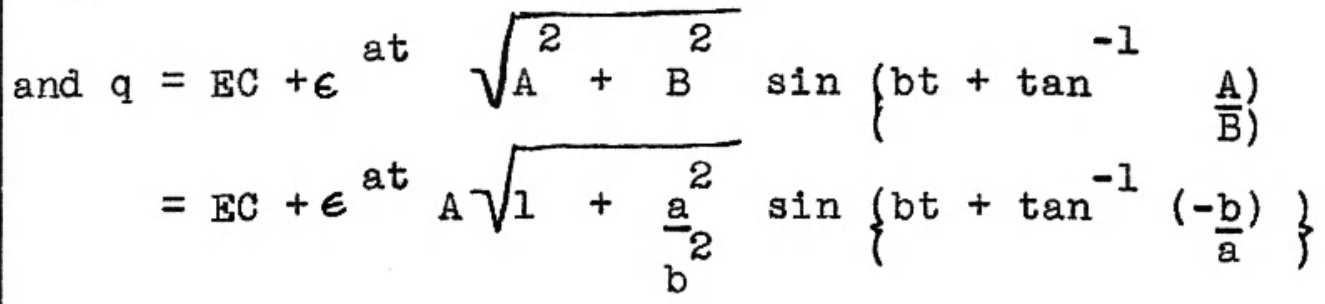

$$
\begin{aligned}
& =E C-E C \epsilon^{a t} \sqrt{1+\frac{a^{2}}{b^{2}}} \sin \left\{b t+\tan ^{-1}\left(-\frac{b}{a}\right)\right\} \\
& =E C-E C \quad \frac{2 \sqrt{L C}}{\sqrt{4 L C-r^{2} C^{2}}} \in \frac{r t}{2 L} \sin \left\{\sqrt{\frac{4 L C-r^{2}}{2 L C}} t+t^{-1}\right. \\
& \sqrt{\left.\frac{4 L C-r^{2}}{r C}\right\}}
\end{aligned}
$$

and from equation (7) the expression for the current becomes

$$
i=\frac{2 E C}{\sqrt{4 L C-r C}} \epsilon^{\frac{-R T}{2 L}} \sin \sqrt{\frac{4 L C-r^{2} C^{2}}{2 L C}} t
$$

The particular integral, i.e., the term representing the steady state for the equation

$$
E_{m} \sin (W t+\alpha)=r \frac{d q}{d t}+I \frac{d q}{d t^{2}}+\frac{q}{c}
$$

may be found as follows:

Under steady conditions, the voltage drop across the circuit will be made up of three parts, namely: $r i=r \frac{d q}{d t}$ 
the voltage drop across the resistance, $I \frac{d i}{d t}=L \frac{d q}{2}$, the dt

voltage drop across the self-inductance, and $\frac{\mathrm{q}}{\mathrm{C}}=\frac{\mathrm{l}}{\mathrm{C}} \int \mathrm{idt}$, the voltage drop across the capacitance. If $r, L$ and $C$ are constant, both the charge and current must vary sinusoidally with time when the impressed electromotive force is sinusoidal.

Assume that the charge is given by

$$
q=Q_{m} \sin \left(W t+\alpha+\theta^{\prime}\right) \quad \text { (10) } 134
$$

When $\theta^{\prime}$ is the phase angle of the charge with respect to the impressed electromotive force, then

$$
\begin{aligned}
E_{m} & \sin (W t+\mathcal{L}) \\
= & r \frac{d q}{d t}+L \frac{d^{2} q}{d t^{2}}+\frac{q}{C} \\
= & W r Q_{m} \cos \left(W t+\alpha+\theta^{\prime}\right)-W^{2} L Q_{m} \sin \left(W t+\alpha+\theta^{\prime}\right) \\
+ & \frac{1}{C} Q_{m} \sin \left(W t+\alpha+\theta^{\prime}\right)
\end{aligned}
$$

Since the cosine of an angle is equal to the sine of ninety degrees plus the angle, equation (II) may be written in the following form: 
$\left(W t+\alpha+\theta^{\prime}\right)+\frac{1}{C} Q_{m} \sin \left(W t+\alpha+\theta^{\prime}\right)$

The vectors corresponding to the terms of equation (12) are plotted in figure 1 for the instant of time

$$
t=-\frac{\alpha+\theta}{W} \text {. }
$$

This instant of time was chosen because it puts the vector representing the charge along the axis of reference.

From figure 1 it is obvious that

$$
\begin{aligned}
E_{m} & =\sqrt{\left(W r Q_{m}\right)^{2}+\left(\frac{1}{c} Q_{m}-W^{2} L Q_{m}\right)^{2}} \\
& =W Q_{m} \sqrt{r+\left(\frac{1}{W C}-W L\right)}
\end{aligned}
$$

and $Q_{m}=\frac{E_{m}}{W \sqrt{r^{2}+\left(\frac{1}{W C}-W L\right)^{2}}}$

$$
\begin{aligned}
\tan \theta^{\prime} & =\frac{W r Q_{m}}{\frac{1}{C} Q_{m}-W^{2} L Q_{m}} \\
& =\frac{r}{\frac{I}{W C}-W L}
\end{aligned}
$$




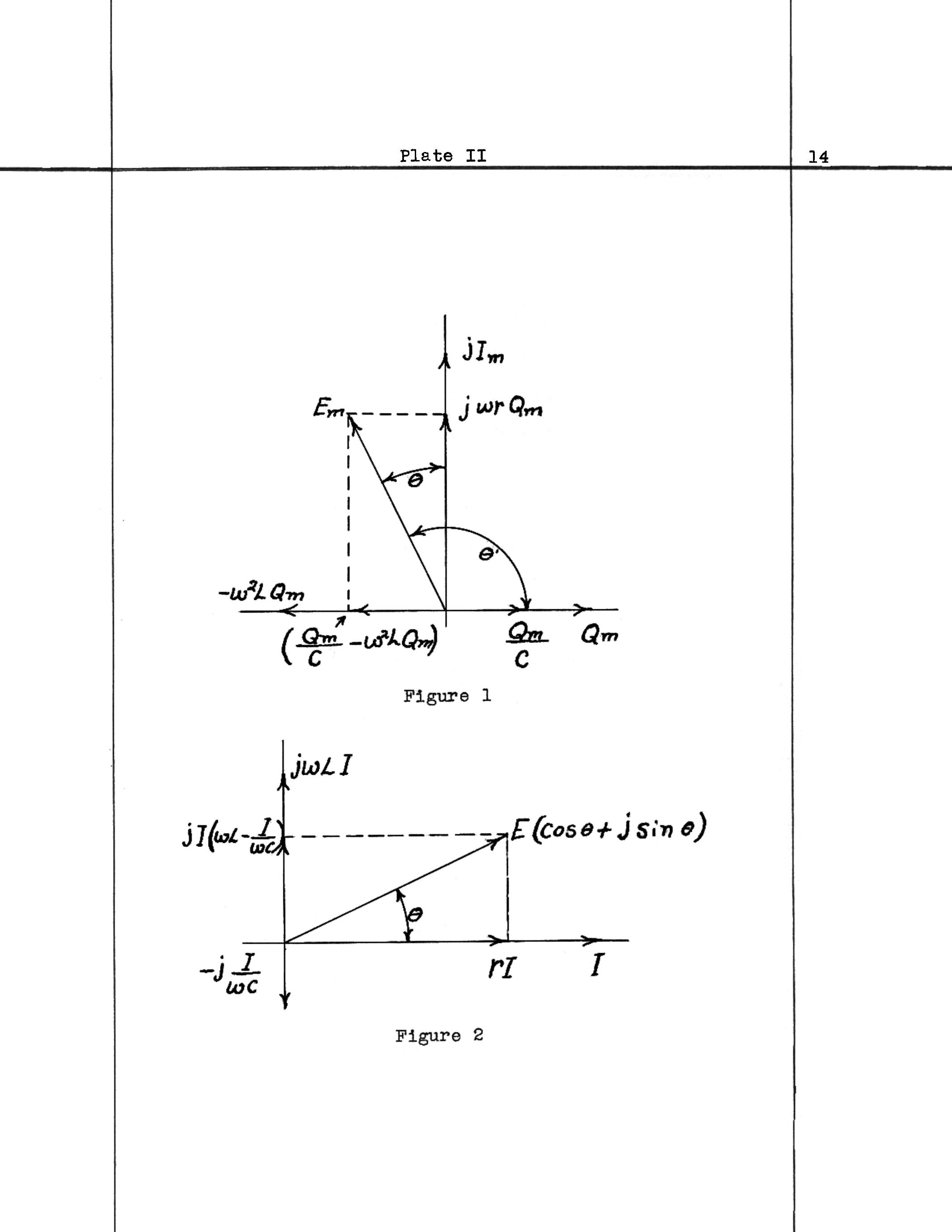


The charge lags the voltage, $\mathrm{E}_{\mathrm{m}} \mathrm{sin}(\mathrm{Wt}+\alpha)$, impressed across the circuit, by the angle $\theta^{\prime}$. The angle $\theta^{\prime}$, therefore, represents the lag of the charge behind the impressed voltage or the lead of the impressed voltage with respect to the charge. When $\frac{1}{\overline{W C}}$ is greater than WL, $\tan \theta^{\prime}$ is positive and $\theta^{\prime}$ is less than 90 degrees. When $\frac{1}{W C}$ is less than WL, $\tan \theta^{\prime}$ is negative and the charge lags the impressed voltage by more than 90 degrees but less than 180 degrees. If $r$ is zero, then $\theta^{\prime}$ is also zero. In this case the charge is in phase with the voltage impressed across the circuit, which, under this condition, is the same as the voltage across the condenser.

The expression for the charge when steady conditions have been reached is therefore

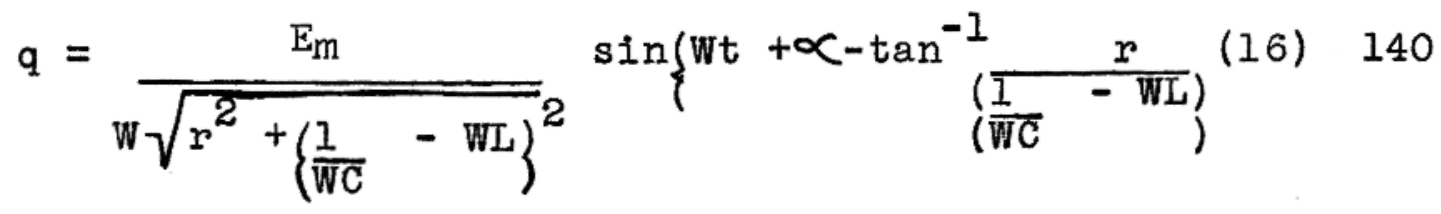

The $\theta^{\prime}=\tan ^{-1} \frac{r}{\left.\frac{(1}{(W C}-W L\right)}$ in equation (16) is a lag

since equation (16) is an equation for the charge in terms of the impressed electromotive force. The charge lags the impressed electromotive force, $E_{m} \sin (W t+\alpha)$, by the angle $\theta^{\prime}$. 


$$
\begin{aligned}
& \text { Since } 1=\frac{d q}{d t} \text {, the current under steady condition is }
\end{aligned}
$$

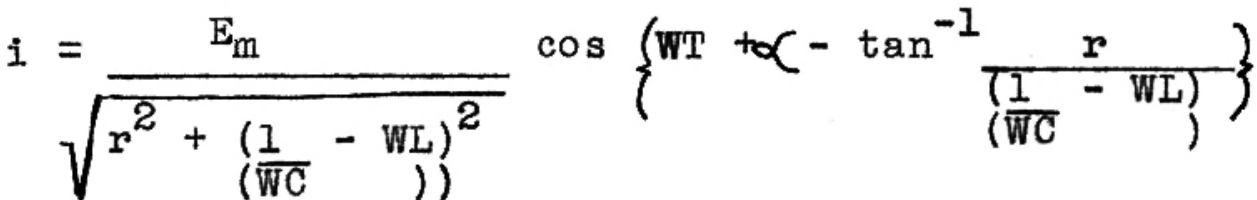

$$
\begin{aligned}
& i=\frac{E_{m}}{\sqrt{r^{2}+\left(\frac{1}{W C}-W L\right)}} \\
& \sin \begin{cases}W t+\alpha-\tan ^{-1} & \frac{r}{\left(\frac{I-W L}{}-W^{-1}\right)} \\
& \left(\overline{W C}^{\circ}\right)\end{cases}
\end{aligned}
$$

The current, therefore, leads the charge by 90 degrees. The vector representing the current is plotted in figure 2.

Let $\theta$ be the angle which the current makes with the impressed electromotive force, $\mathbf{E}_{\mathrm{m}} \cdot$ Then

$$
\begin{aligned}
\theta & =\theta^{\prime}-90^{\circ} \\
-\theta & =\tan ^{-1}\left\{\frac{\frac{1}{C} Q_{m}-W^{2} L Q_{m}}{W r Q_{m}}\right\} \\
& =\tan ^{-1} \frac{W L-\frac{1}{W C}}{r}
\end{aligned}
$$

Equation (17) may, therefore, be written

$$
i=\frac{E_{m}}{\sqrt{r^{2}+\left(W L-\frac{1}{W C}\right)^{2}}} \sin \left\{w t+\alpha-\tan ^{-1}\left\{\frac{W L-\frac{1}{W C}}{r}\right\}(18) \quad 142\right.
$$


The signs of WL and $\frac{1}{W C}$ in the radical

$\sqrt{r^{2}+\left(W L-\frac{1}{W C}\right)^{2}}$, of equation (18), are reversed in the expression (WL $-\frac{1}{W C)}$ to make it correspond to the like term in the expression for the tangent of the angle $\theta$. This can can be done because the term (WL $-\frac{1}{W C}$ ) is squared and changing its sign does not alter its squared value.

The complete solution for equation (2) for charge is given by adding equation (16) to equation (5).

$$
\begin{aligned}
& q=\Psi+u=A_{1} \epsilon^{a_{1} t}+A_{2} \epsilon^{a}{ }^{t}+\frac{E_{m}}{W \sqrt{r^{2}+\left(\frac{1}{W C}-W L\right)^{2}}} \sin \{W t+\alpha \\
& \left.-\tan ^{-1} \frac{r}{\left(\frac{1-W L}{\left(\overline{W C}^{-}\right.}\right)}\right\}
\end{aligned}
$$

And the complete solution of equation (2) for current as given by adding equation (18) to equation (5.')

$$
\begin{aligned}
& 1=Y+u=A_{1} a_{1} e^{a_{1} t}+A_{2} a_{2} e^{a_{2} t+\frac{E_{m}}{2^{2}}} \sin \\
& \left\{w t+\alpha-\tan ^{-1} \frac{\left(w L-\frac{1}{W C}\right)}{r}\right\} \\
& \overline{\mathrm{WC}} \text { ) } \\
& \text { (20) } 144
\end{aligned}
$$


After a brief interval of time, the transient terms in equations (19) and (20) become sensibly zero for circuits with constants ordinarily met in practice and the charge and current then become simple harmonic functions of time.

The maximum value of the current under steady condition is found by dividing the maximum value of the impressed electromotive force by

$$
\sqrt{r^{2}+\left(w L-\frac{1}{w C}\right)^{2}}
$$

The current lags the voltage by an angle whose tangent is $\left.\frac{\left(W L-\frac{1}{W C}\right)}{r}\right)$

When $\frac{1}{W C}$ is greater than WL, the angle $\theta$ becomes negative and is then equivalent to an angle of lead. In this case the current actually leads the electromotive force impressed on the circuit by an angle $\theta$. When $\frac{1}{\mathrm{WC}}$ is less than WL, the angle $\theta$ is positive and is actually an angle of lag. In this case the current lags the voltage Impressed on the circuit. 
Since the effective value of a sinusoidal wave is equal to its maximum value divided by the square root of two, it is evident that the effective value of the current is found by dividing the effective value of the voltage by

$$
\sqrt{r^{2}+\left(W L-\frac{1}{W C}\right)^{2}}
$$

This current leads or lags the impressed voltage accordingly as $\frac{1}{W C}$ is greater or less than WL.

$$
\begin{gathered}
I=\frac{E}{{\sqrt{r^{2}+\left(W L-\frac{I}{W C}\right)^{2}}}^{2}}=\frac{E}{\frac{W}{Z}} \\
\left.\theta=\tan ^{-1} \quad\left(\frac{\left(W L-\frac{1}{W C}\right.}{r}\right)\right)
\end{gathered}
$$

(22) 146

The quantity $\mathrm{z}=\sqrt{r^{2}+\left(W L-\frac{1}{W C}\right)^{2}}$ (23) is called the impedance and is measured in ohms. It is constant only when resistance, self-inductance, capacitance and frequency are constant. It may increase or decrease with an increase in frequency depending on the relative values of WL and $\frac{1}{\overline{W C}}$. The expression WL $=x_{L}$ is the inductive reactance and the expression $-\frac{1}{W C}=x_{C}$ is the capacitive reactance. 
$\left(W L-\frac{1}{W C}\right)=x_{L}+x_{C}=x_{0}$ is the resultant reactance. It should be noted that inductive reactance is always positive while capacitive reactance is always negative. The resultant reactance may be elther positive or negative depending upon the relative values of $x_{L}$ and $x_{C}$. Inductive reactance always increases with increase of frequency. Capacitive reactance always decreases with increase of frequency. 
When WL $=\frac{1}{W C}, x_{0}=0$ and the current is given by $\frac{E}{r}$ and is in phase with the impressed electromotive force. Under this condition the circuit is said to be in resonance.

The addition of a sine and cosine curve may also be obtained by use of trigonometry as worked out by Prof. E. R. Lyon in the following manner. (See Plate III).

The mean square of the effective current of the coupled circuit when the capacity is varied between the limits, $\mathrm{C}_{1}$ and $\mathrm{C}_{2}$, which are equally removed on either side of the resonant value $\mathrm{C}_{r}$, will now be determined.

$$
I=\frac{E}{\sqrt{r^{2}+\left(W L-\frac{1}{W C}\right)^{2}}}
$$

Let $I^{2}=\bar{y} ; \quad W=2 \pi n$

$$
\begin{aligned}
& \overline{\mathrm{y}}=\frac{\mathrm{s}^{2}}{\mathrm{C}_{2}-\mathrm{C}_{1}} \int_{\mathrm{C}_{1}}^{\mathrm{C}_{2}} \frac{\mathrm{dc}}{\mathrm{r}^{2}+\left(W L-\frac{1}{\mathrm{WC}}\right)^{2}} \\
& \overline{\mathrm{y}}=\frac{\mathrm{E}^{2}}{\mathrm{C}_{2}-\mathrm{C}_{1}} \int_{\mathrm{C}_{1}}^{\mathrm{C}_{2}} \frac{\mathrm{c}^{2} \mathrm{dc}}{\mathrm{R}^{2} \mathrm{c}^{2}+\left(W L C-\frac{1}{W}\right)^{2}}
\end{aligned}
$$




$$
\bar{y}=\frac{w^{2} E^{2}}{c_{2}-c_{1}} \int_{c_{1}}^{c_{2}} \frac{c^{2} d c}{w^{2} R^{2} c^{2}+\left(w^{2} L c-1\right)^{2}}
$$

Let $c=x ; \propto=w^{2} R^{2} ; B=w^{2} L$

$$
\begin{aligned}
& \bar{y}=\frac{w_{E}^{2}}{x_{2}-x_{1}} \int_{x_{1}}^{x_{2}} \frac{x^{2} d x}{x^{2}+(B x-1)^{2}} \\
& \overline{\bar{y}} \\
& \left.\frac{w^{2} E_{2}^{2}-x_{1}}{x_{1}}\right)^{x^{2}+B^{2} x^{2}-2 B x+1}
\end{aligned}
$$

Let $a=1 ; b=-2 B=-2 W^{2} L ; K=\alpha+B^{2}=W^{2} R^{2}+W^{4} L^{2}$

$$
\overline{\mathrm{y}} \frac{\mathrm{w}^{2} \mathrm{E}^{2}}{\mathrm{x}_{2}-\mathrm{x}_{1}} \int_{\mathrm{x}_{1}}^{\mathrm{x}_{2}} \frac{\mathrm{x}^{2} \mathrm{dx}}{\mathrm{a}+\mathrm{bx}+\mathrm{kx}}
$$

Let $x=a+b x+k x^{2}$

$$
\begin{gathered}
q=4 a K-b^{2}=4 W^{2} R^{2}+4 w^{4} L^{2}-4 w^{4} L^{2}=4 w^{2} R^{2} \\
q>0
\end{gathered}
$$




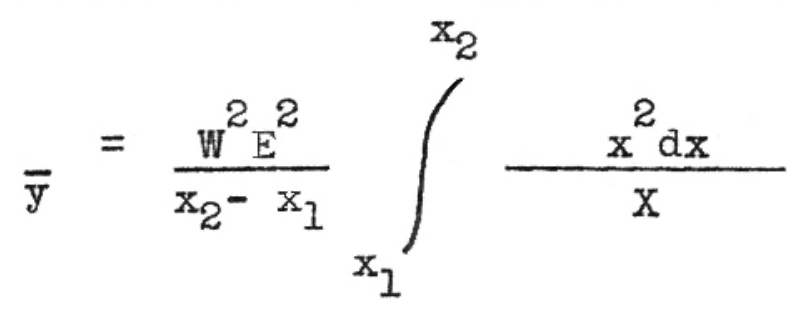

Then from Peirce the following is obtained (Page 10, eq. 75 and 67).

$$
\begin{aligned}
& \bar{y}=\left(\frac{W^{2} E^{2}}{\left.x_{2}-x_{1}\right)}\right)\left(\frac{x}{K}-\frac{b \log x}{2 K^{2}}\right)_{x_{1}}^{x_{2}}+ \\
& \frac{\left(w^{2} E^{2}\right)}{\left(x_{2}-x_{1}\right)}\left(\frac{b^{2}-2 a K}{2 K^{2}}\right)\left\{\frac{2}{\sqrt{q}} \tan ^{-1}\left(\frac{2 K x+b}{(\sqrt{q})}\right)\right\}_{x_{1}}^{x_{2}}
\end{aligned}
$$$$
\bar{y}=I^{2}=\frac{\left(W_{E}^{2}\right)}{\left(C_{2}-C_{1}\right)}\left\{\frac{C}{\left(W^{2} R^{2}+W^{4} L^{2}\right.}+\right.
$$$$
\frac{W^{2} L \log \left(1-2 W^{2} L C+W^{2} R^{2}+W^{4} L^{2} C^{2}\right.}{\left(W^{2} R^{2}+W^{4} L^{2}\right)^{2}}\left\{C^{C_{2}}+\right.
$$

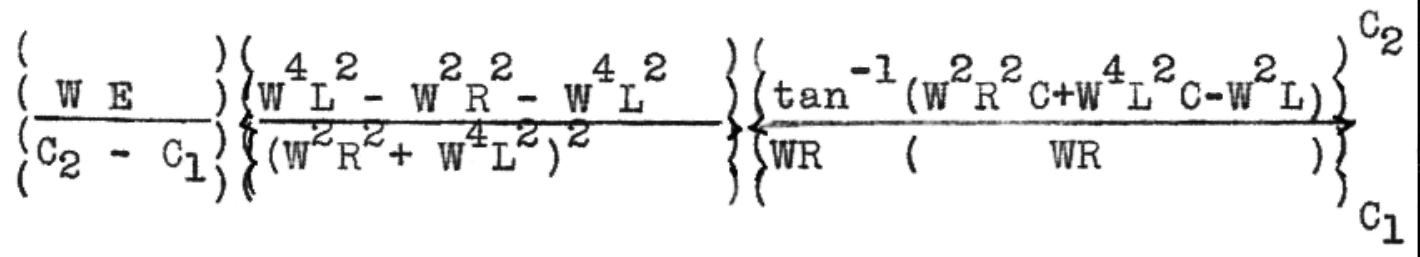


$I^{2}=\frac{W^{2} E^{2}}{W^{2} R^{2}+W^{4} L}+\left\{\frac{W^{2} E^{2}}{C_{2}-C_{I}}\left\{\frac{\left(W^{2} L \log \left\{\left(\frac{\left.1-W^{2} L C\right)^{2}+W^{2} R^{2} C^{2}}{2}\right\}\right)\right.}{\left(W^{2}+W^{4}\right)^{2}}\right\}\right.$

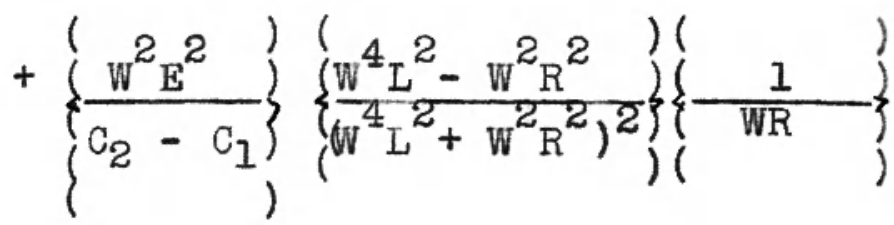

$\left\{\tan ^{-1}\left(\frac{W R^{2} C+W^{3} L^{2} C-W L}{R}\right)\right\}_{C_{1}}^{C_{2}}$

$I^{2}=\frac{E^{2}}{R^{2}+W^{2} L^{2}}+\left\{\frac{L}{\left(C_{2}-C_{I}\right.}\right\}\left\{\frac{E^{2}}{\left(R^{2}+W^{2} L^{2}\right)^{2}}\right\}$

$\left\{\log \left(\frac{\left.C_{2}\right)^{2}}{C_{1}}+\frac{\left(1-w^{2} L C_{2}\right)^{2}}{W^{2} R^{2} C_{2}^{2}}-\frac{\left(1-w^{2} L C_{1}\right)^{2}}{w_{R}^{2} C_{1}^{2}}\right\}\right.$

$+\left\{\frac{W E^{2}}{R\left(C_{2}-C_{1}\right)}\right\}\left\{\frac{W^{2} L^{2}-R^{2}}{\left.W^{2} L^{2}+R^{2}\right)^{2}}\right\}\left\{\frac{\pi}{2}-\tan ^{-1}\right.$ $\left(\frac{R}{W{ }^{2} C_{2}+W^{3} L^{2} C_{1}-W L}\right)+\tan ^{-1}\left(\frac{R}{W R^{2} c_{1}+W^{3} L^{2} C_{1}-W L}\right)$ 


$$
\begin{aligned}
I^{2}=\frac{E^{2}}{Z_{2}} & +\ldots \ldots \ldots \\
I^{2}=\frac{E_{2}^{2}}{Z^{2}} & \left.+\left\{\frac{2 R L E}{2 R Z^{4}\left(C_{2}-C_{1}\right)}\right\}\left\{\log \left(\frac{C_{2}}{C_{1}}\right)^{2}\right\}\right) \\
& +\frac{\pi W E^{2}\left(W^{2} L^{2}-R^{2}\right)}{2 R Z^{4}\left(C_{2}-C_{1}\right)}
\end{aligned}
$$

in as much as the neglected part of the preceding equation is negligible if $R^{2}<L / C$,

$$
\begin{aligned}
& I^{2}=\left\{\frac{E^{2}}{Z^{2}}\right\}+\frac{\pi E^{2}\left(W^{2} L^{2}-R^{2}\right)}{2 R Z^{4}\left(C_{2}-C_{1}\right)} ; \\
& I^{2} \propto \frac{1}{C_{2}-C_{1}}
\end{aligned}
$$$$
\text { when } C_{2}=c_{r}+\Delta C ; C_{1}=c_{r}-\Delta c \text {. }
$$

This is true only when working on the curve on both sides of the maximum value and very near the top. 


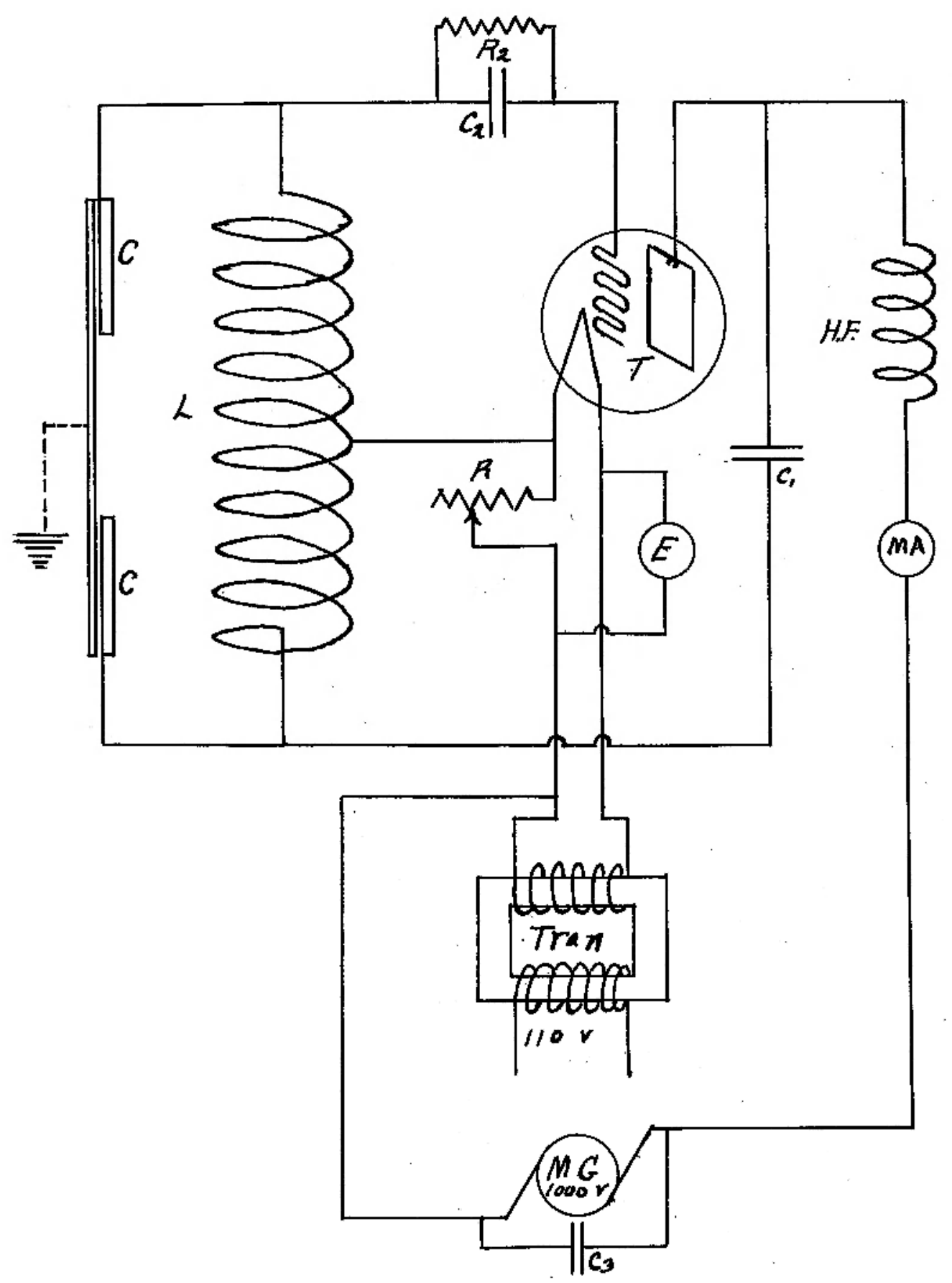




\section{CONSTRUCTION OF THE APPARATUS}

The apparatus used was assembled from standard parts found in the department or was made in the shop.

The Hartley oscillator that was constructed for use as the source of power, or the sending set, required the construction of a special induction coil (L) and a variable condenser (C); the rest of it being standard parts used in assembling a 50-Watt broadcast transmitter and furnished by the Radio Corporation of America.

The various parts required to assemble a 50-Watt oscillator are listed by letter, corresponding to the letters used in the circuit diagram Plate IV, in the following list.

$\mathrm{I}=$ Special inductance coil (10 turns \#6 Cu wire)

$\mathrm{C}=$ Special variable condenser

$T=50$-Watt Model UV 203 Radiatron

$\mathrm{R}=$ Filament rheostat Model PT 53\% (R.C.A.)

$\mathrm{R}_{2}=50,000 \mathrm{ohm}$ transmitting grid leak U.P. 1718 (R.C.A.) $\mathrm{C}_{1} \mathrm{C}_{2}=$ Faradon Condensers, Model Uc 1014 (.002 $\mu f$, 3,000 volts)

$\mathrm{H} . \mathrm{F} .=$ About $200 \mu \mathrm{H}$ Choke

$\mathrm{E}=0-15$ voltmeter 
MA $=\theta-500$ Millimeter

$\mathrm{Mg}=1,000$ volt D. C. Motor Generator set

$c_{3}=1 \mu f_{\text {Moulded Condenser }}$

Tran $=110-12$ volt filament transformer

$\mathrm{x}$ = Tube socket UT 541 (R.C.A.) (Not in diagram)

In addition to the above, there are required two bakelite panel boards, about 14 inches by 14 inches and onefourth inch thick, to serve as front and back panels. The base is made of hard wood that has been boiled in a special insulating compound. Paraffin or beeswax may be used. The base is 12 inches by 14 inches and seven-eighths inch thick. Six large binding posts and three feet of No.6 bare copper wire are also required.

The inductance (I) consists of ten turns of No.6 copper or aluminum wire wound in a coil, four and one-half inches in diameter and equally spaced over a distance of twelve inches. The coil is mounted on a strip of hard rubber or bakelite as shown in figure 3, Plate $V$, and this is mounted on the front and top of the instrument panel. The ends of the wire were threaded and fitted with large brass binding posts. Large wire was used not only to hold the shape of 
the coil better and make it more rigid, but mainly to reduce the resistance to as near zero as possible.

The inductance of this coil may be calculated by using the formula of Nagaoka as found in any textbook on radio. The table of constants used in this paper was taken from "Principles of Radio Communication" by J. H. Morecroft, pages 193 and 194 .

$$
I=4 \quad 2 \mathrm{R}^{2} \mathrm{~N}_{1}^{2} I \mathrm{~K} \quad \mathrm{~cm}
$$

in which

$$
\begin{aligned}
I= & \text { inductance in } \mathrm{cm} \\
\mathrm{R}= & \text { radius of coil to center of wire, in } \mathrm{cm}=6 \mathrm{~cm} \\
\mathrm{~N}_{1}= & \text { number of turns of wire per cm of length } \\
I= & \text { length of winding in } \mathrm{cm}=30 \mathrm{~cm} \\
\mathrm{~K}= & \text { constant }=\frac{2 \mathrm{R}}{\mathrm{I}}=\text { ratio of coil diameter to the } \\
& \text { coil length }
\end{aligned}
$$

The value of (I) inductance obtained by the above formula is only approximate as it was designed for the turns close together.

The values of $(K)$ constant have been worked out by H. Nagaoka and are given in the following table. 


\begin{tabular}{|c|c|c|c|c|c|c|c|c|c|c|c|}
\hline$\frac{2 R}{1}$ & : & $\mathrm{K}$ & $:$ & $\frac{2 R}{I}:$ & $\underline{K}$ & $\frac{2 R}{1}:$ & $\mathrm{K}$ & $: \frac{2 R}{1}:$ & $\mathrm{K}$ & $\frac{2 R}{1}:$ & $\mathrm{K}$ \\
\hline 0.00 & : & 1.000 & & $.40:$ & 0.850 & $: 0.80:$ & 0.735 & $: 1.60:$ & 0.580 & $: 5.00:$ & 0.320 \\
\hline .05 & : & .979 & : & $.45:$ & .834 & : .85: & .723 & :1.80: & .551 & $: 6.00:$ & .285 \\
\hline .10 & : & . 959 & : & $.50:$ & .818 & : $.90:$ & .711 & $: 2.00:$ & .526 & $: 7.00:$ & .258 \\
\hline .15 & : & .939 & : & $.55:$ & .803 & : .95: & .700 & $: 2.50:$ & .472 & $: 8.00:$ & .237 \\
\hline .20 & : & .920 & : & $.60:$ & .789 & $: 1.00:$ & .688 & $: 3.00:$ & .429 & $: 9.00:$ & .219 \\
\hline .25 & : & . 902 & : & $.65:$ & .775 & $: 1.10:$ & .667 & $: 3.50:$ & .394 & $10.00:$ & .203 \\
\hline .30 & : & .884 & : & $.70:$ & .761 & $: 1.20:$ & . 648 & $: 4.00:$ & .365 & $:--$ : & ---- \\
\hline .35 & : & .867 & : & $.75:$ & .748 & $: 1.40:$ & . 611 & $: 4.50:$ & . 341 & $:--:$ & --- \\
\hline
\end{tabular}



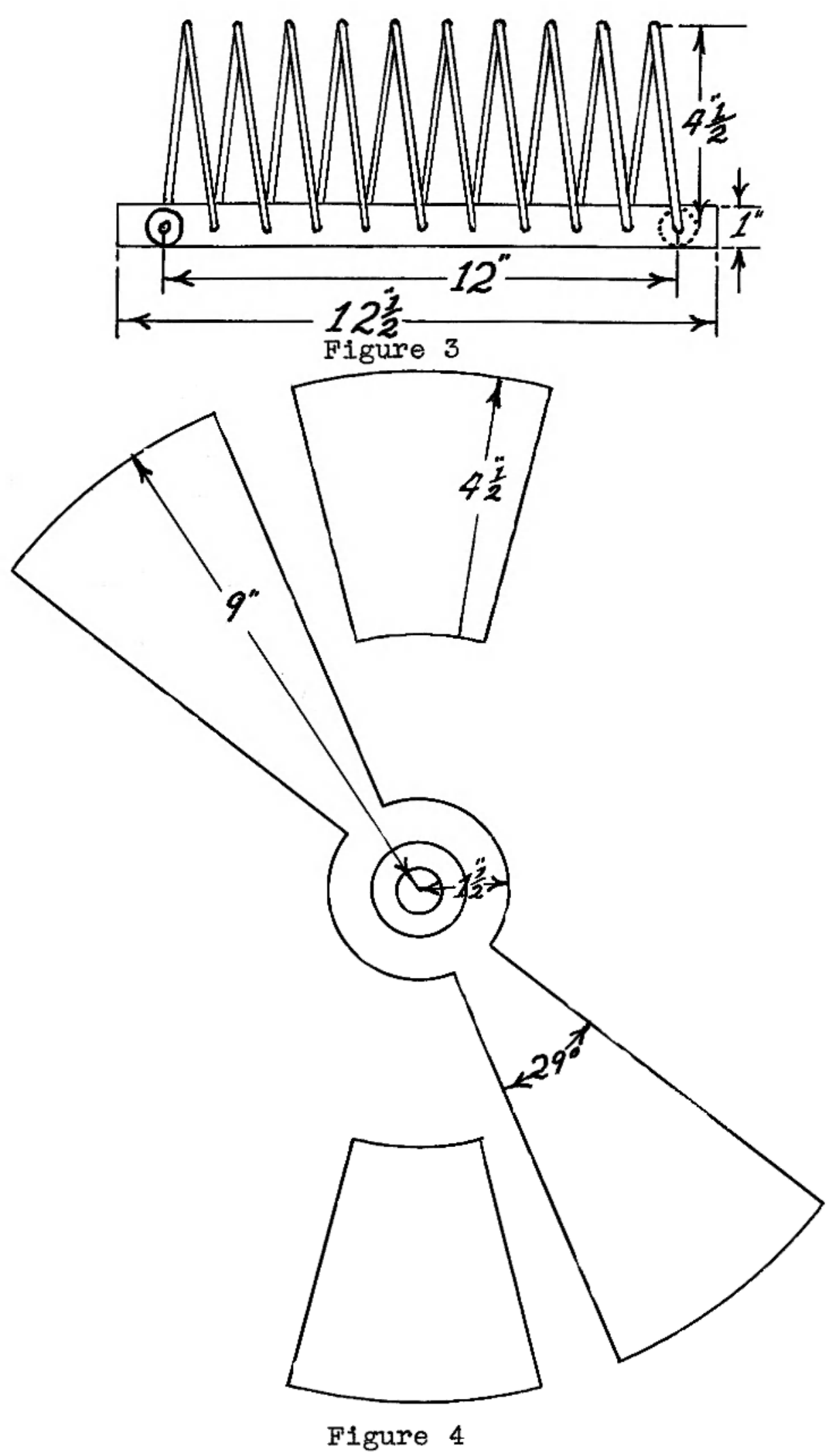


\section{Condenser Construction}

The variable condenser is constructed of two stationary and one movable plate cut from aluminum, onefourth inch thick, and arranged as shown in figure 4, Plate V. The stationary plates are mounted in the same surface and $180^{\circ}$ to each other. They are mounted with a three-point screw support and held in place with a spring on the reverse side. This enables the capacity to be changed by regulation of the air gap and makes alinement of the plates less difficult.

The rotary plate is of one piece, cut as shown, and mounted on an iron shaft which is held by a brass bearing at the rear top of the back board. A hard rubber rod, one and one-half to four feet long, is used to turn the plate. This insulating rod is necessary because of the high voltage used and the length is required to place the operator as far away as possible, in order to reduce the body capacity effect.

The capacity may be found by applying the ordinary formula found in any electrical textbook, $c=\frac{\mathrm{KA}}{4 \pi \mathrm{d}}$. 
Where $\mathrm{C}$ is the capacity expressed in centimeters,

$K=$ the specific inductive capacity of the dielectric

$A=$ area of one side of one plate in square centimeters

$\lambda=$ separation of plates in $\mathrm{cm}$. 
This formula is approximate only. Since the two stationary plates make up a series condenser, the total capacity will be only one-half that obtained by using the formula above.

To express centimeters in micromicrofarads, divide by nine-tenths.

$$
\frac{\mathrm{cm}}{.9}=\mu \mu f
$$

If one millimeter is allowed between plates as the safe sparking distance for the 1,000 volts plate circuit, then the maximum capacity of the condenser will be

$$
\mathrm{c}=\frac{\mathrm{AK}}{4 \pi \mathrm{d}}=\frac{98.04 \times 1}{12.5664 \times \mathrm{I}}=76.4 \mathrm{~cm}
$$

Since the two stationary plates are in series and are the same size, the maximum capacity will be just one-half that obtained above.

$$
\frac{76.4}{2}=38.2 \mathrm{~cm}
$$

or

$$
\frac{38.2}{.9}=42.4 \mu \mu \mathrm{f} .=.0424 \times 10^{-3} \mu \mathrm{f} .
$$

When the reactance of a circuit is zero, we find the resonant frequency from the following 


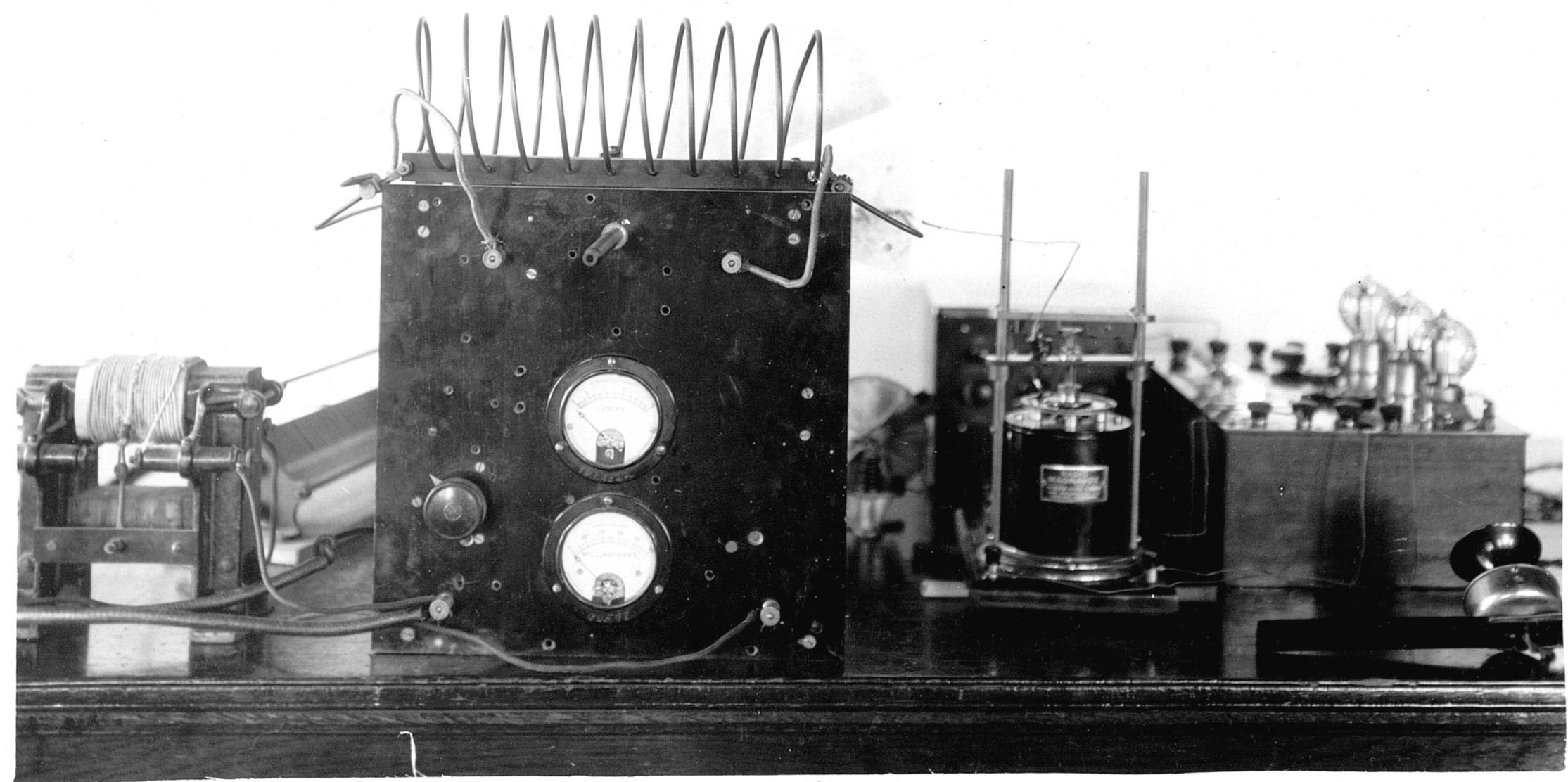


so

$$
\begin{aligned}
& 2 \pi \mathrm{fL}=\frac{1}{2 \pi \mathrm{fC}} \\
& \mathrm{f}=\frac{1}{2 \pi} \sqrt{\frac{1}{L C}}
\end{aligned}
$$

When $I$ is expressed in henries, $C$ in farads, the frequency $f$ will be cycles per second, or

$$
f=\frac{1,000}{2 \pi \sqrt{\mathrm{LC}}}
$$

when $\mathrm{C}$ is expressed in microfarads

The critical frequency is fixed by the product of I $\mathrm{X} C$, and the sharpness of the resonance curve is determined by the resistance in the circuit. The nearer the resistance approaches zero, the sharper the curve.

\section{Receiving Circuit}

A wave meter, or receiving set, was designed with the same constants as that used in the oscillator. It consists of an inductance in series with a special variable condenser and a thermo-junction galvanometer, as shown in wiring diagram. (Fig. 5, Plate $x$ ).

The inductance (I) was constructed to have the same value as that used in the Hartley oscillator, the dimensions being the same in both cases. 
The condenser $\mathrm{c}_{s}$ is specially built to give a variable value of capacity that could be controlled by the frequencies within the range of the voice.

It is built up from a large type radio Magnavox loud speaking type $R 2$ Model $B$, as the base of construction. The metal diaphram was replaced by a sheet of high grade thin mica to which the moving coil was attached. The upper side of the mica carries a thin sheet of tin foil that acts as one of the plates. Above this plate is mounted a brass disk that may be raised or lowered with a screw adjustment to vary the air space between the two. Both plates are coated with a very thin layer of some insulating varnish or shellac to prevent short circuiting. in case they touch each other. See Plate IX for details of construction and dimensions. 
Plate VII

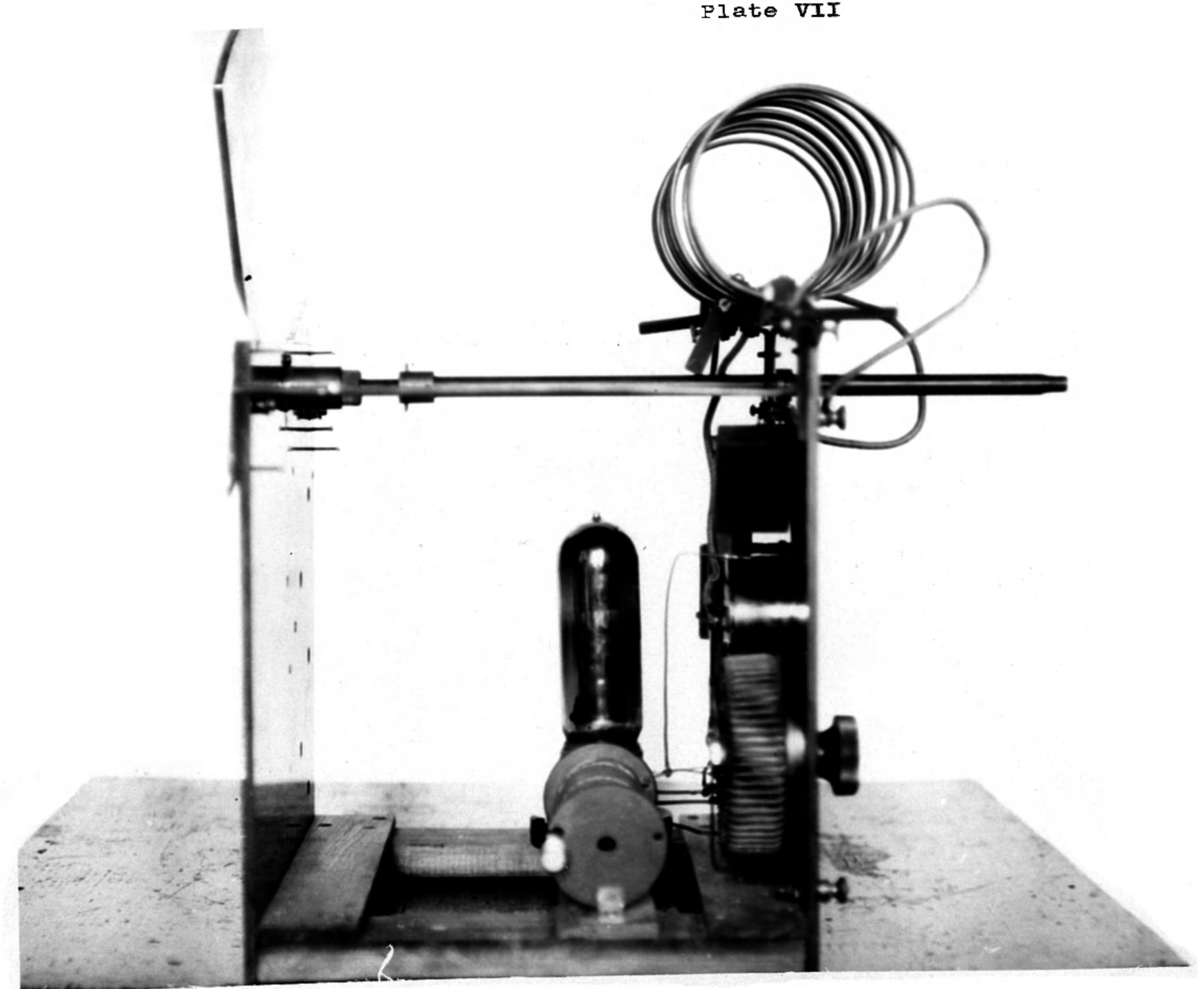




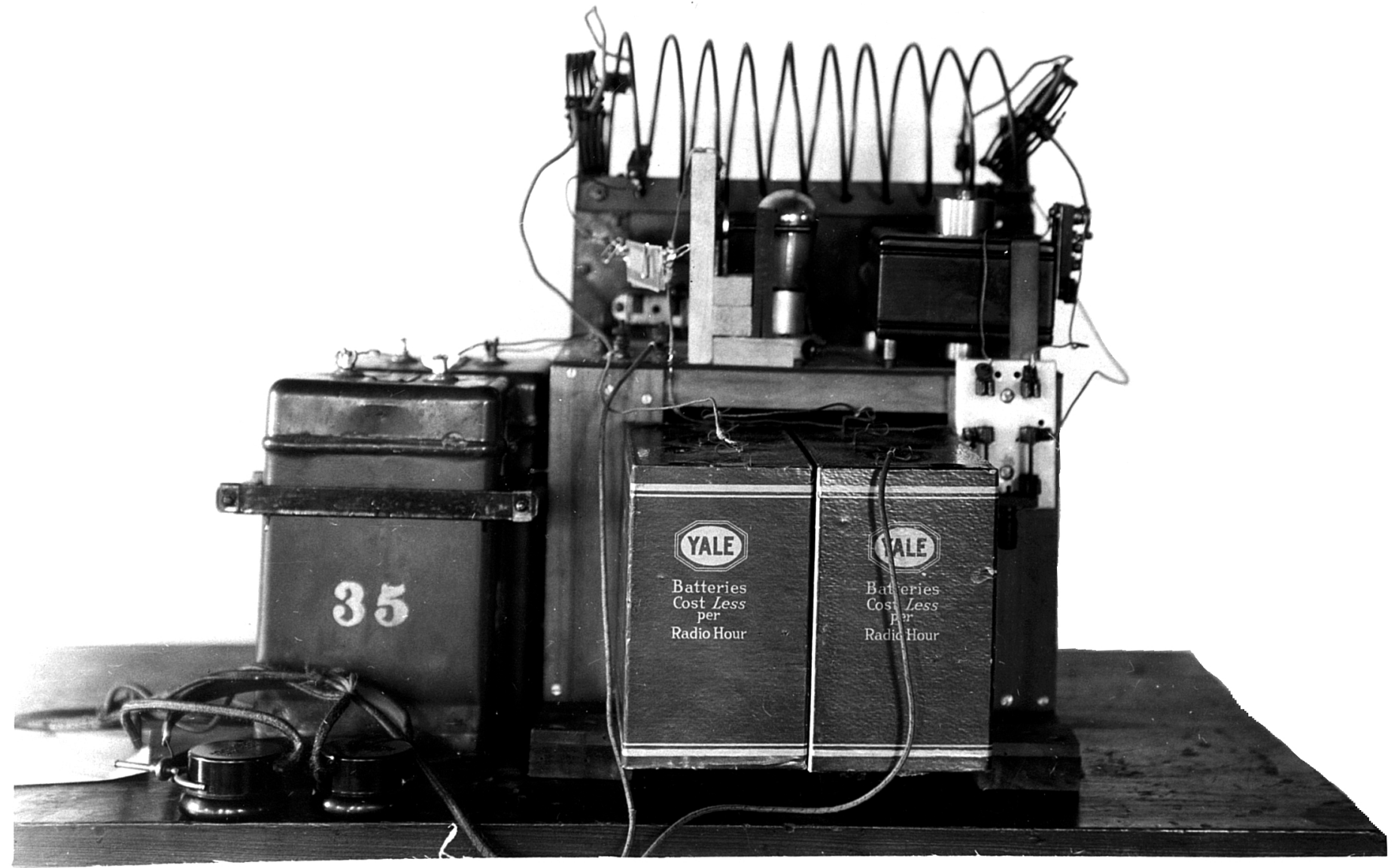


The capacity, when the condenser is not vibrating, may be obtained by the usual formula for a single plate condenser, as stated before. But the variable capacity could only be obtained by measuring the current and using formula as worked out under theory. 
Plate IX

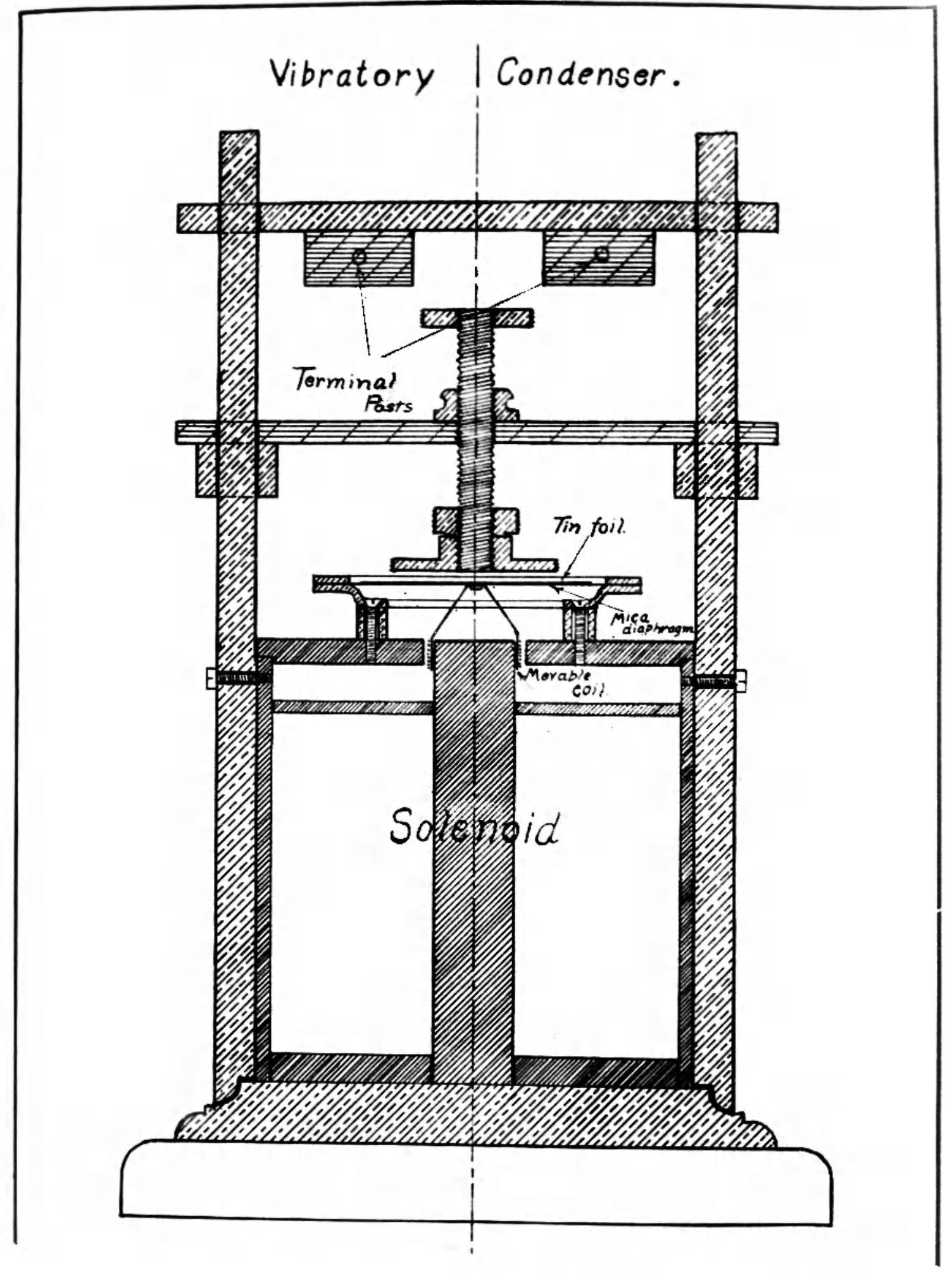




\section{RESULTS AND CONCLUSIONS}

The first tests made were to determine whether or not the vibrating condenser, constructed as described, would affect the current flowing in the receiving circuit.

The circuit was arranged as shown in figure 7 and tuned to the wave-length of the Hartley oscillator and adjusted until about half-scale deflection was obtained on the thermo-junction galvanometer. Then the condenser was set in vibration, and the reading of the galvanometer changed, showing that the set was thrown out of resonance.

The condenser was made to vibrate by connecting the input terminals to the output of a three-tube power amplifier box which was connected to a 110 volts, 60 cycles, current through a potentiometer and modulation transformer as shown in figure

The voltage on the input was then varied by means of the potentiometer, and the reading of the galvanometer was found to change, being greatest when maximum voltage was used and least when minimum was applied. This shows that the capacity of the condenser varies inversely as the applied voltage as stated by Wente and Crandall in the Physical Review for July, 1917, and June, 1918. 


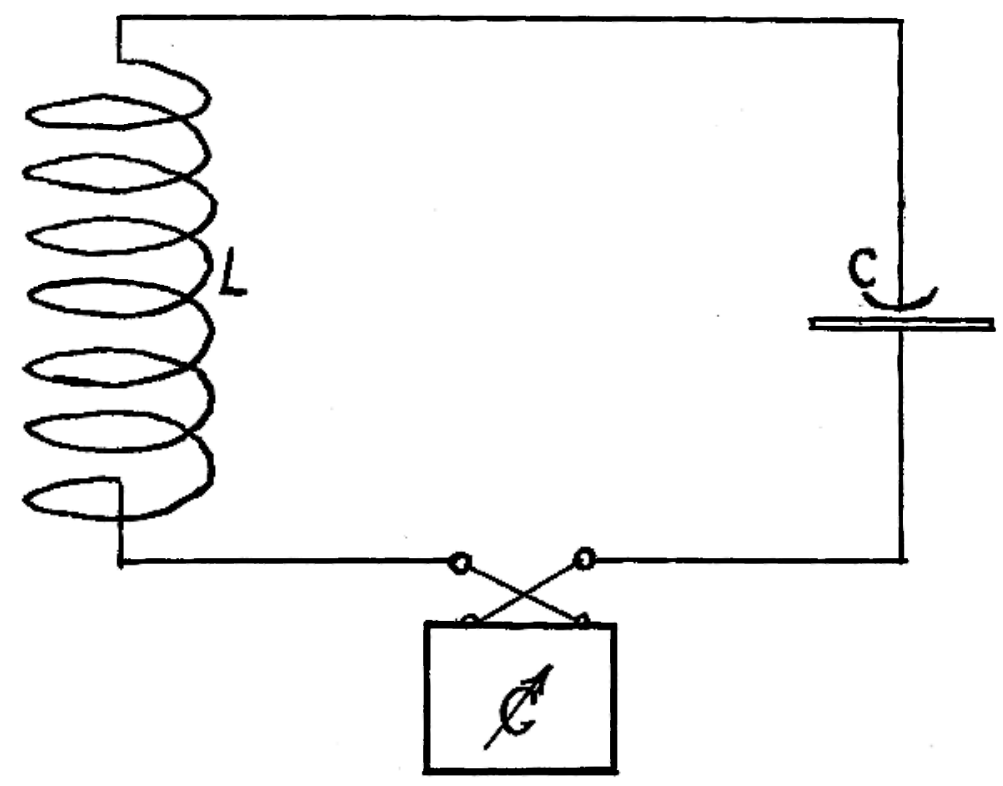

Figure 5

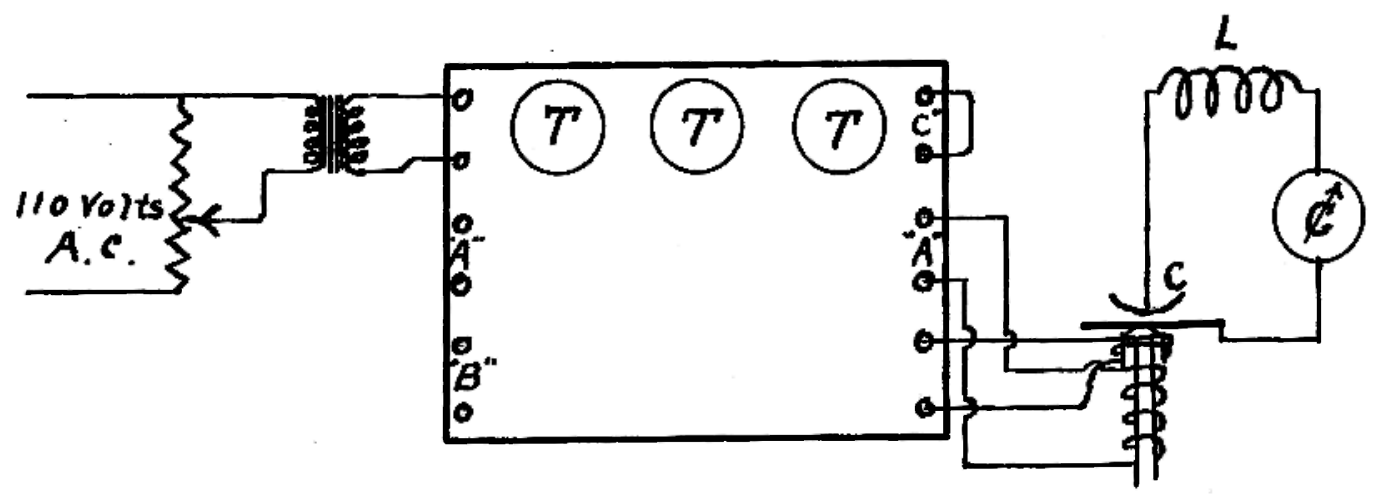

Figure 6 

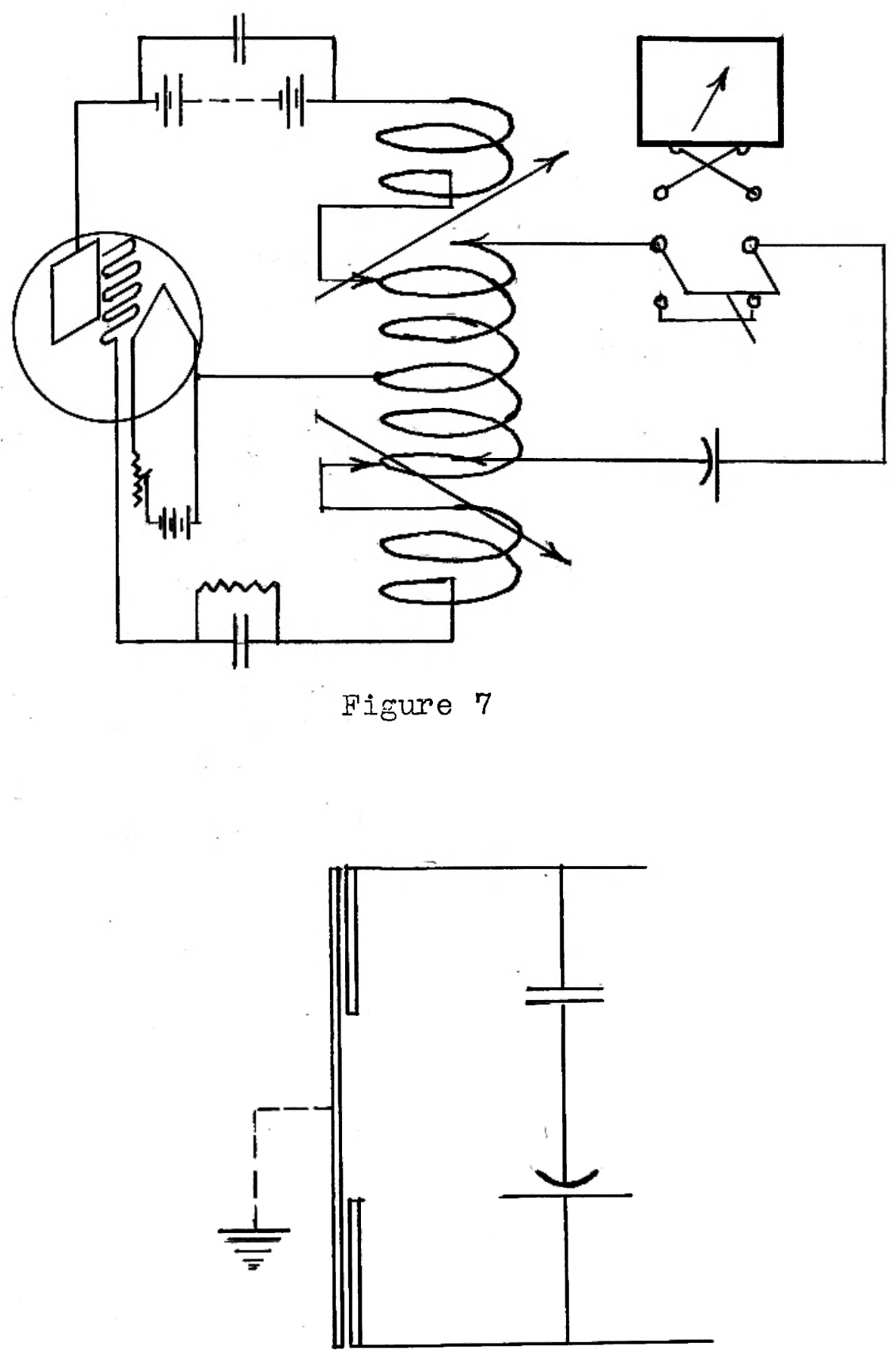

Figure 8 
When a continuous vocal tone was used instead of the alternating current, the galvanometer reading was also caused to drop off.

These tests showed that the resonance condition of the circuit could be changed by vibration of the condenser and that the amount of the change varied in proportion to the amplitude of the impressed voltage.

The receiving set was then connected with a vacuum tube as in figure 7 and the vibratory condenser was replaced by a small variable condenser of approximately the same capacity.

The vibratory condenser was connected in series with a small transmitting condenser and the two placed in parallel with the condenser on the transmitting set. (See Fig. 8 ). Energy was supplied to the vibrator as before.

The wave length of the transmitter was then measured by the Lecher wire method as outlined on page 170, experiment No. 102, of Experimental Radio by Ramsey. Resonance was found to be sharpest at about eight meters. The corresponding frequency is $\frac{300,000,000}{8}=37.5 \times 10^{6}$ oscillations per second. 
The receiving set was then tuned to this wave-length, and it was found that the condenser acted as a modulator controlled by the voice.

In the ordinary type of modulation the amplitude of the oscillating current that flows in the oscillating circuit is directly controlled by the amplitude of the audio frequency current. Figure 9 shows the wave-form of an unmodulated oscillator, giving a current of constant amplitude and constant frequency. When an audio-wave, figure 10 is impressed upon the microphone of the modulator circuit, the plate voltage of the oscillator tube is made to vary as the audio frequency varies. This causes the outgoing, or transmitted wave, to vary likewise, and a wave of varying amplitude is obtained. Figure 11 shows the modulated transmitted wave. 


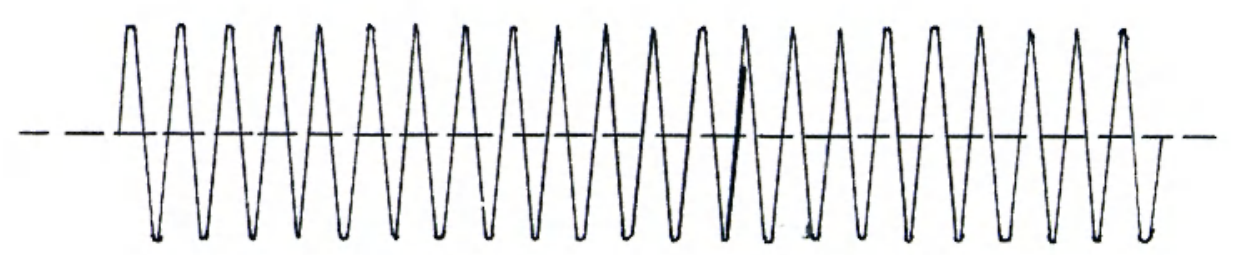

Figure 9
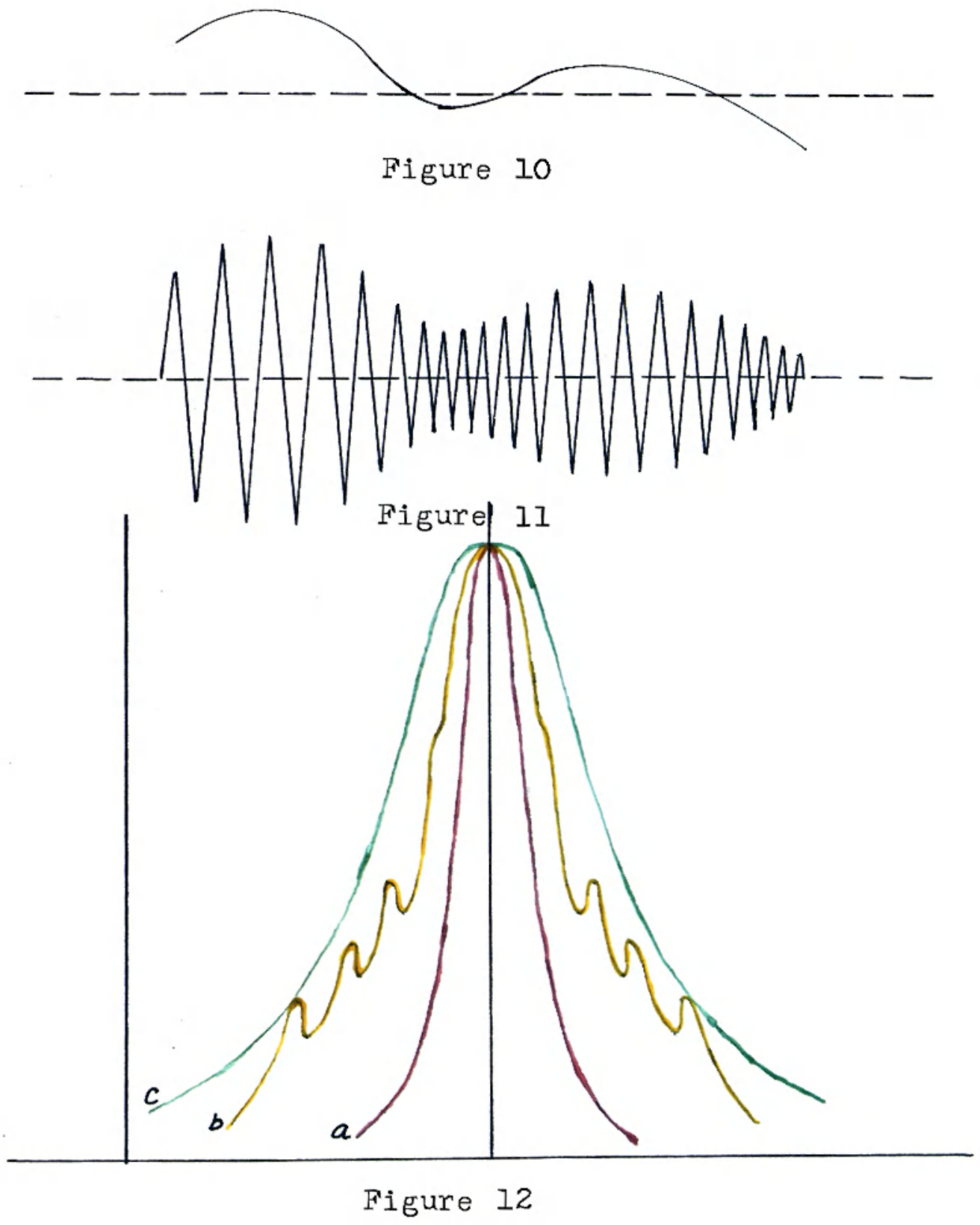


\section{Plate XIII}

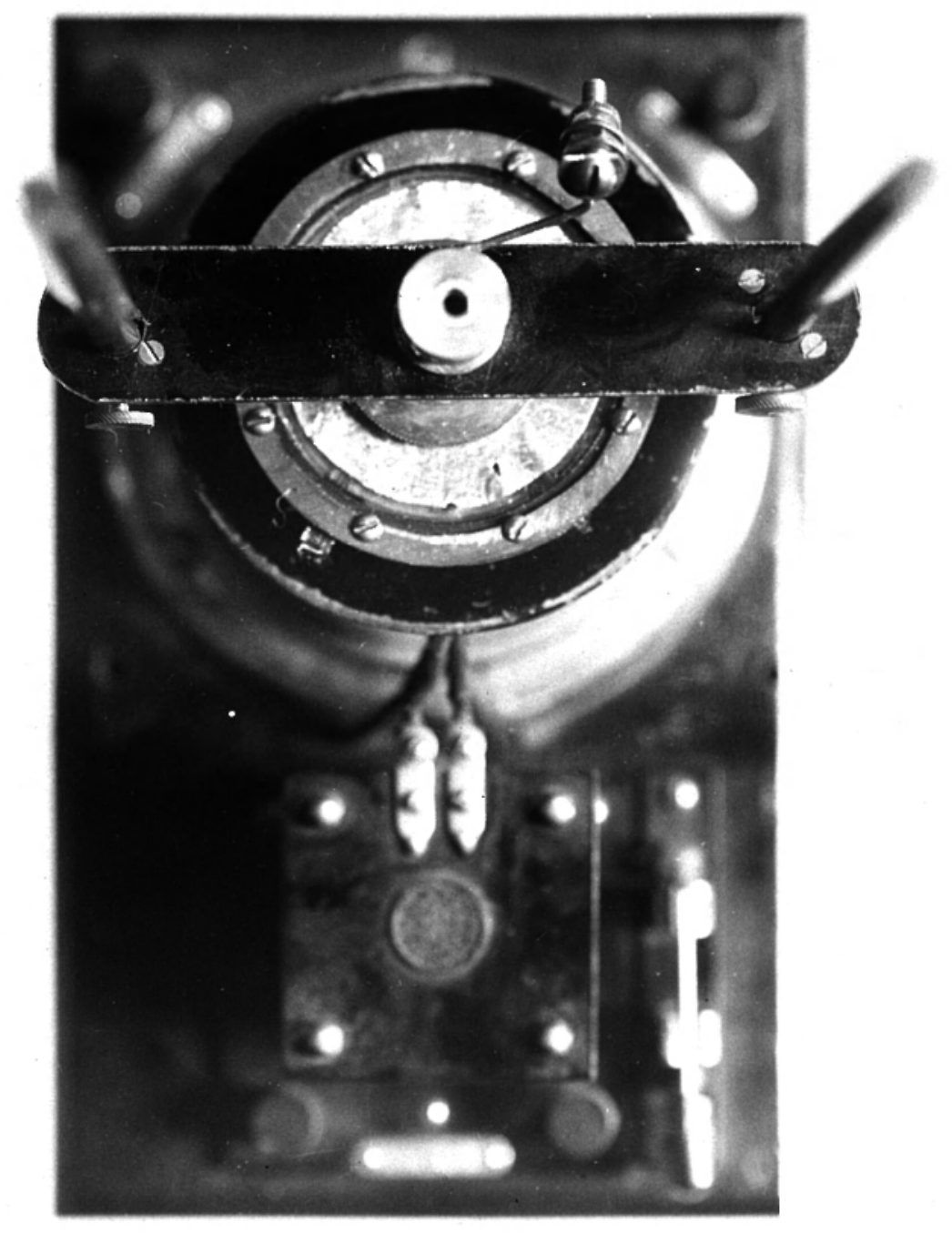


This method of combining radio-frequency current with audio-frequency control of its amplitude yields resultant frequencies that are, respectively, the sum and the difference of the radio and audio frequencies. Therefore, a broadcast wave and its side-bands are, together, about five kilocycles broad. Consequently, a receiving set that responds to all of the side-bands must be tuned somewhat broad. If curve a (red) in figure 12 represents the resonance curve of the unmodulated transmitter, curve $\underline{b}$ (yellow) will be that of the modulated or transmitted wave resulting from the previously described beats-producing, or heterodyning effect of the modulation. If the receiving set is to respond to this broadcast wave, it must be tuned broadly enough to cover the field range, as shown by curve $\underline{\text { (green). }}$

In the type of modulation used in the experiments described by this thesis, the amplitude of the current going into the oscillator remains constant. Modulation is obtained by variation of the capacity in the oscillating circuit. The capacity change results in a broadcast wave of variable frequency. The radio frequency changes in amount, depending upon the change of capacity of the 
51

vibrating condenser, which in turn, depends upon the amplitude of the audio-frequency vibration of the vibrating condenser. The audio-frequency is represented in the final wave-train, merely, by the cyclically and periodically progressing system of alterations of the radio frequency, and not by any simple function of the frequency of the radio current existing at any particular time. Figures 13, 14, 15, show this type of modulation. 


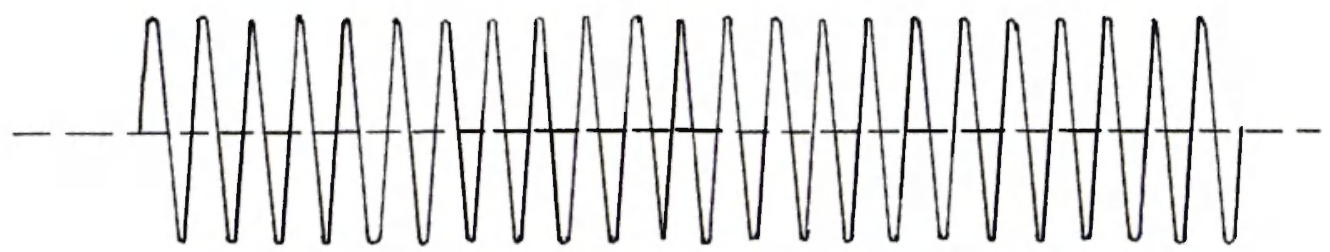

Figure 13

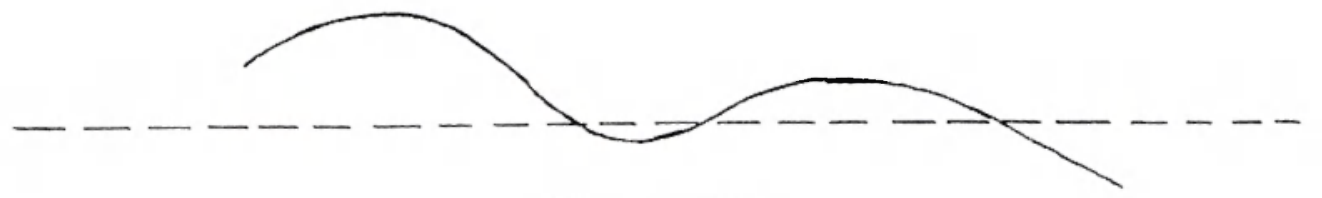

Figure 14
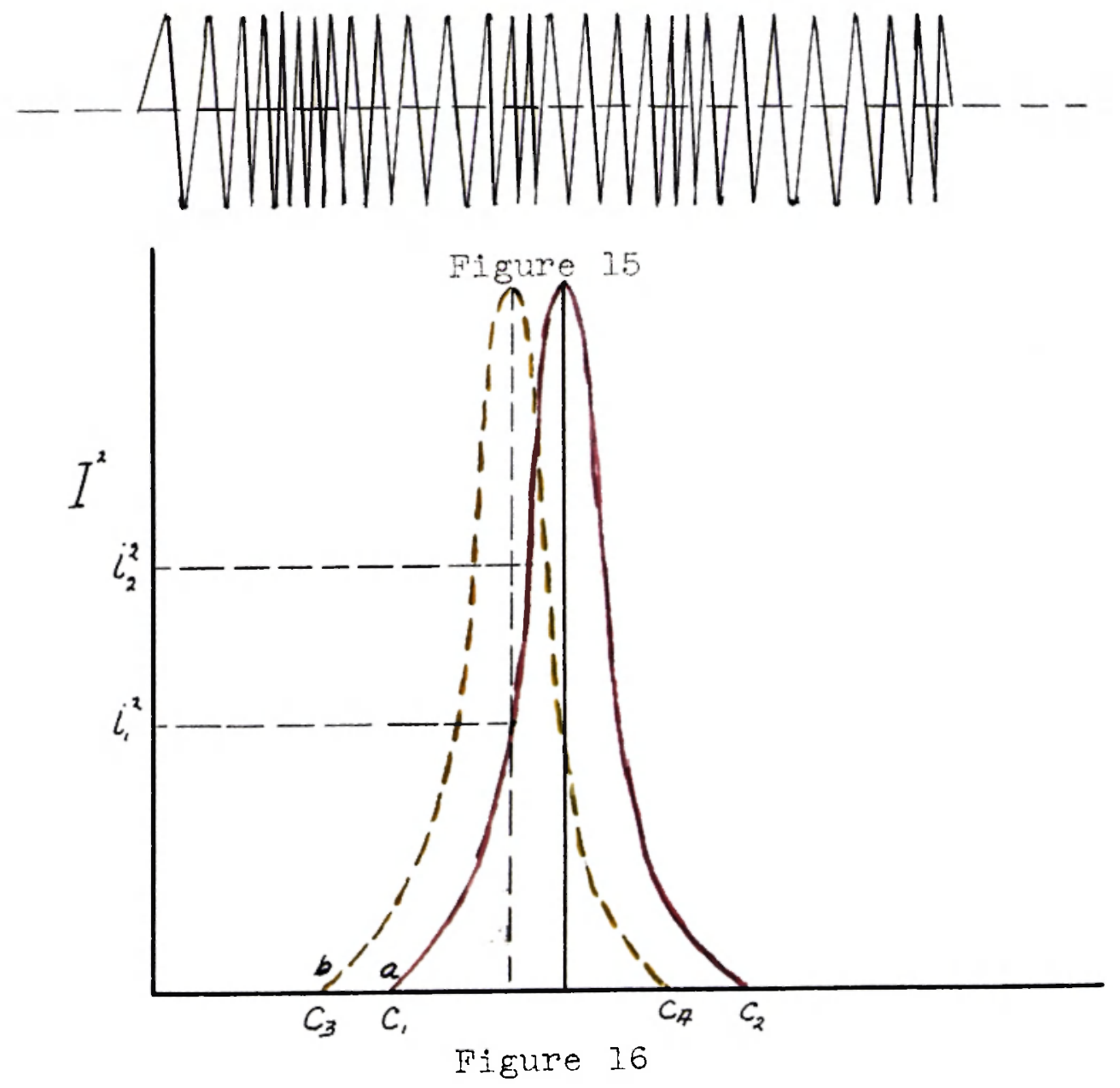
If curve a (red) of figure 16 represents the

resonance curve of the transmitter at constant frequency, it is seen that any change in frequency, caused by a capacity change, will produce a shift in the resonance curve, as shown by $\underline{b}$ (yellow). Thus, the current in a receiving set, tuned to resonance with the unmodulated transmitter wave, will vary as the transmitted frequency changes in response to the audio-frequency alteration of the capacity in the transmitter's circuit. $I_{m}^{2}$ is the resonance current at capacities $C_{1}$ and $C_{2} \cdot I^{2}{ }_{1}$ is the current in the receiver when the capacity of the transmitter has changed to $\mathrm{c}_{3}$ and $\mathrm{c}_{4}$. This illustrates how the current varies as the transmitted frequency is varied.

This makes possible very fine tuning of the receiving set, because the shift of the operating point on the resonance curve, due to this small change of radio-frequency that has been impressed by the amplitude of modulation, can be made to yield a large current change for extremely small radio frequency changes if the decrement of the receiving circuit is extremely small. 
I wish to express my appreciation to my major instructor, Prof. E. R. Lyon, for outlining and directing this work, to Prof. J. O. Hamilton for making possible the pictures of the apparatus, and to all others who have helped to make this a very pleasant and profitable year. 


\section{SUPPLEMENTARY READING}

Nothing bearing directly upon this method of modulation is to be found in the literature. Topics indirectly related are --

\section{Crandall, I. B.}

Air Damped Vibrating system.

Physical Review. June, 1918.

Wente, E. C.

Electrostatic Transmitters.

Physical Review. July, 1917. 